\title{
AN APPLICATION OF THE METHOD OF THE ACCELERATION POTENTIAL*
}

\author{
BY \\ J. LEHNER (Cornell University) AND C. MARK (University of Manitoba)**
}

In this paper Prandtl's theory of the acceleration potential is used, in conjunction with conformal mapping, in order to determine the pressure distribution on a symmetrical control surface consisting of a fin and flap separated by a gap of finite width, under the assumption of a steady irrotational flow of an incompressible perfect fluid. The method used is essentially that by which $\mathrm{M}$. A. Biot ${ }^{1}$ recently derived the wellknown formulae for lift and moment of a symmetrical airfoil with flap in a remarkably simple manner. The present problem is considerably more complicated than that of a single airfoil; but it is still possible to obtain formulae in closed form. In the case where the gap between fin and flap is large in proportion to the chord of either, the formulae obtained here for the pressure distribution on the fin, or on the flap, do not differ materially from those used for a single symmetrical airfoil.

For treatments of this or related problems by the classical velocity-potential method, see I. Flügge-Lotz and I. Ginzel, Die ebene Strömung um ein geknicktes Profil mit Spalt, Ingenieur-Archiv 11, 268-292 (1940), which also contains references to earlier studies. Flügge-Lotz and Ginzel do not restrict themselves to the symmetrical case (fin and flap of equal length) as is done in this paper. The method by which they obtain the complex velocity potential is essentially the same as the one we use to derive the acceleration potential. They compute the pressure distribution for an unsymmetrical split wing rather than the total lift and moment, so that a comparison of their numerical results with ours is not practicable.

A paper by Kutta in the Sitzber. Bayerische Akad. of 1911 considers the special case in which the two airfoils have the same angle of attack. Our results agree with his if we make the identification $\sin 2 \alpha=2 \alpha$, where $\alpha$ is the angle of attack.

The first part of the paper gives a description of the methods and results; while some details of the mathematical methods used are given in the second part.

\section{General Description of Methods and Results}

1. The acceleration potential. The equation of motion of an incompressible perfect fluid of density $\rho$ is

$$
\rho \bar{a}=-\operatorname{grad} p,
$$

where $\bar{a}$ denotes the acceleration vector, and $p$ the pressure. According to Prandtl, ${ }^{2}$

* Received Jan. 18, 1943.

** This paper was prepared at the suggestion of Professor W. Prager while the authors were participants in the Program of Advanced Instruction and Research in Mechanics at Brown University, Summer 1942. The authors are greatly indebted to Dr. L. Bers for valuable suggestions.

${ }^{1}$ M. A. Biot, Some Simplified Methods in Airfoil Theory, Journal of the Aeronautical Sciences, 9, 185-190 (1942).

${ }^{2}$ L. Prandtl, Beitrag zur Theorie der tragenden Fläche, Zeitschrift f. angew. Math. u. Mech., 16, 360361 (1936). 
the function

$$
\varphi=\frac{-p}{\rho}
$$

may be called the acceleration potential, since $\bar{a}=\operatorname{grad} \varphi$.

We shall consider the steady irrotational plane flow around a symmetrical control surface consisting of a fin and flap separated by a finite gap. Taking the axis of $x$ parallel to the velocity $U$ which prevails at an infinite distance from the control surface, we write the components of the velocity vector $\bar{q}$ as

$$
q_{x}=U+u, \quad q_{y}=v .
$$

In the case of a thin profile with a small angle of attack, the terms $u$ and $v$ can be assumed to be small compared with the velocity $U$ of the undisturbed stream.

For a steady flow, the components of the acceleration vector are

$$
a_{x}=q_{x} \frac{\partial q_{x}}{\partial x}+q_{y} \frac{\partial q_{x}}{\partial y}, \quad a_{y}=q_{x} \frac{\partial q_{y}}{\partial x}+q_{y} \frac{\partial q_{y}}{\partial y} .
$$

Introducing the acceleration potential $\varphi$ on the left sides and the expressions (1.3) on the right sides of these relations, and neglecting terms of the second order in $u$ and $v$, we obtain

$$
\frac{\partial \varphi}{\partial x}=U \frac{\partial u}{\partial x}, \quad \frac{\partial \varphi}{\partial y}=U \frac{\partial v}{\partial x} .
$$

According to the condition of incompressibility, $\partial u / \partial x+\partial v / \partial y=0$, we have $\partial u / \partial x=$ $-\partial v / \partial y$. Substituting this in the first of our equations gives

$$
\frac{\partial \varphi}{\partial x}=-U \frac{\partial v}{\partial y}, \quad \frac{\partial \varphi}{\partial y}=U \frac{\partial v}{\partial x} .
$$

Elimination of $v$ between these two equations leads to

$$
\frac{\partial^{2} \varphi}{\partial x^{2}}+\frac{\partial^{2} \varphi}{\partial y^{2}}=0
$$

The acceleration potential $\varphi$ is thus seen to satisfy the Laplace equation and, consequently, can be taken as the real part of an analytic function, $f(x+i y)=\varphi(x, y)$ $+i \psi(x, y)$, of the complex variable $x+i y$. M. A. Biot has shown that the conjugate function of the acceleration potential, $\psi(x, y)$, also has an immediate physical significance. Indeed, from (1.4) and the well-known Cauchy-Riemann relations, $\partial \varphi / \partial x=\partial \psi / \partial y, \partial \varphi / \partial y=-\partial \psi / \partial x$, it follows that the function $\psi$ may be defined so as to equal $-U v$.

In the rest of this section we shall speak of only one airfoil on the understanding that what is said applies equally to the fin and the flap.

The undisturbed flow $U$ along the $x$-axis involves a normal velocity $+U \alpha$ at the surface of a thin straight airfoil having the small angle of attack $\alpha$. The boundary condition of tangential flow on the surface will be satisfied by a function giving the velocity $-U \alpha$ normal to the surface. As is usual in the linear approximation, this boundary condition is applied at the $x$-axis instead of at the surface; so that we now take a part of the $x$-axis to represent the airfoil (see Fig. 1). We have, then, $v=-U \alpha$; and hence,

$$
\psi=-U v=U^{2} \alpha
$$


that is, $\psi$ is constant on the surface of the airfoil.

The problem of finding the pressures, and hence the lift on the airfoil, may now be restated. One seeks an analytic function of the complex variable $x+i y$ the imaginary part of which satisfies the condition (1.6) and, since $\psi=-U v$, vanishes at infinity. The real part of this analytic function may then be used as the acceleration potential from which the pressures may be obtained by (1.2). Taking into account the properties of harmonic functions, the fact that $\psi$ must vanish at infinity in the $z$-plane $(z=x+i y)$ makes it necessary that $\psi$ have at least one singularity. Aerodynamical considerations indicate the leading edge of the airfoil as the obvious location of this singularity, partly by the analogy with the classical thin-wing theory in which the velocity turned out to be infinite at the leading edge. As in the case studied by Biot,

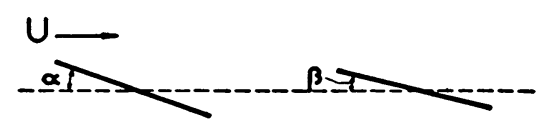

(a)

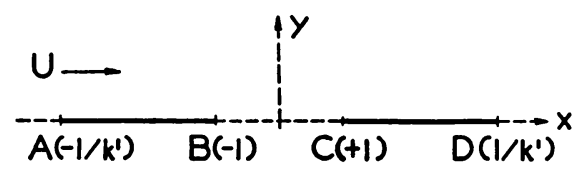

(b): Z-PLANE

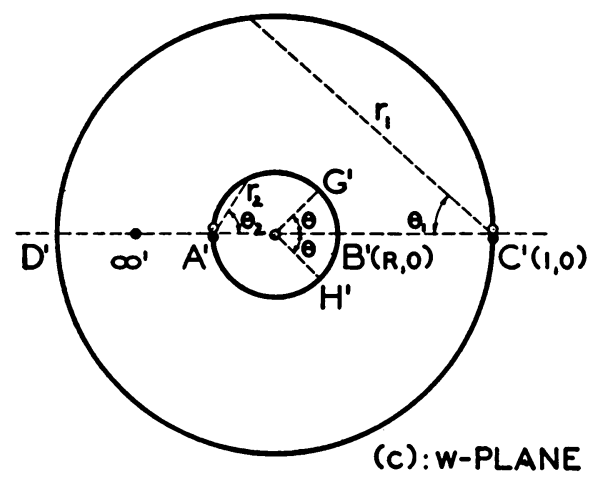

FIG. 1. The actual fin and flap are indicated in (a); the slits used to represent them in (b): it is on these slits that the condition (1.6) is actually met. (c) shows the circles into which (b) is mapped. It is convenient to use temporarily several sets of polar coordinates in the $w$-plane: $(r, \theta)$, with origin at center of circles; $\left(r_{1}, \theta_{1}\right)$, with origin at $C^{\prime} ;\left(r_{2}, \theta_{2}\right)$, with origin at $A^{\prime}$. we assume this singularity to be a source-sink doublet with axis parallel to the $y$-axis.

To simplify further the determination of $\psi$, the segment of the $x$-axis now used to represent the airfoil is mapped conformally in to a circle in the $w$-plane.

2. The mapping. We treat only the symmetrical case in which the fin and flap are of equal length.* Let these be represented by slits along the real axis: the fin from $-1 / k^{\prime}$ to -1 , the flap from 1 to $1 / k^{\prime},\left(0<k^{\prime}<1\right)$. The $z$-plane exterior to the two slits can be mapped into the interior of a circular ring in the $w$-plane, as indicated in Fig. 1 . The radius of the outer circle may be taken as unity, the other radius, $R$, being then fixed. The slits transform into the boundary circles of the ring. The function giving the required mapping can be written in closed form using elliptic functions (see $\S 6$ ).

In the mapping, the upper edge of the slit $A B$ (Fig. 1) goes into the upper semicircle $A^{\prime} B^{\prime}$, the lower edge of the slit into the lower semicircle. Moreover, points such as $G$ and $H$ on $A B, G$ being on the upper edge of the slit and $H$ on the lower edge immediately below $G$, map into points such as $G^{\prime}(R, \theta)$ and $H^{\prime}(R,-\theta)$ for which the values of $\theta$ are equal but opposite in sign. Similarly for $C D$. For uniqueness, we define the coordinate $\theta$ in the $w$-plane so that $|\theta| \leqq \pi$. The point $z=\infty$ maps into $\infty^{\prime}(\sqrt{R}, \pi)$.

* The unsymmetrical case could be handled just as easily at this stage. Any fin-flap arrangement could be mapped into the symmetrical case by a preliminary linear transformation of the type $z^{\prime}=(a z+b) /(c z+d)$; subsequent steps being the same as here. It is in meeting the condition $\psi(\infty)=0$ that the symmetrical case is notably easier to treat; unsymmetrical cases might require numerical handling from that point on. 
The radius of the inner circle (transform of the fin) is given by

$$
R=e^{-2 \pi \mathbf{K}^{\prime} / \mathbf{K}},
$$

where $\mathrm{K}$ and $\mathrm{K}^{\prime}$ are the complete elliptic integrals of the first kind (modulus $k$ ), $k$ being the complementary modulus to $k^{\prime}\left(k^{2}+k^{\prime 2}=1\right)$. The relation between the coordinate $z$ of a point on one of the slits in Fig. 1(b) and the coordinate $\theta$ of the corresponding point in Fig. 1(c) is

$$
z= \pm \frac{1}{\operatorname{dn}(\mathrm{K} \theta / \pi, k)}= \pm \frac{1}{\sqrt{k^{\prime}}} \frac{\vartheta_{4}\left(\frac{1}{2} \theta, \sqrt{R)}\right.}{\vartheta_{3}\left(\frac{1}{2} \theta, \sqrt{R)}\right.}
$$

in which the plus sign holds for $z$ on the flap. The notation of the elliptic and $\vartheta$-functions is that used in Whittaker and Watson, Modern Analysis (Cambridge Univ. Press, 4th Ed., 1927; Chap. XXI and XXII).

3. The potential. The problem now is to find a potential which will vanish at infinity and have a singularity of the doublet type at the leading edges of fin and flap (the points $A^{\prime}$ and $C^{\prime}$ in the $w$-plane), and of which the imaginary part $\psi$ will satisfy condition (1.6) separately for each airfoil; namely,

$$
\psi=U^{2} \alpha \text { on } r=R, \text { and } \psi=U^{2} \beta \text { on } r=1 \text {. }^{*}
$$

In addition, corresponding to the Kutta-Joukowski condition in the classical theory, the acceleration potential must be continuous at the trailing edges of fin and flap.

We place a plane doublet of strength $a_{1}$ at $C^{\prime}$ and one of strength $a_{2}$ at $A^{\prime}$, the axis of each doublet being perpendicular to the real axis. The values of $a_{1}$ and $a_{2}$ will be determined later by (3.1). The complex potential at the point $\xi$, in the plane of a complex variable $u$, due to a doublet of strength $m$ at the point $u=0$ whose axis makes an angle $\eta$ with the real axis is

$$
m \xi^{-1} e^{i \eta}
$$

Thus the potential due to the doublet at $A^{\prime}$, for example, is given by (see Fig. 1(c))

$$
a_{2} r_{2}^{-1} e^{i\left(\pi / 2-\theta_{2}\right)}=a_{2} r_{2}^{-1} \sin \theta_{2}+i a_{2} r_{2}^{-1} \cos \theta_{2} .
$$

The imaginary part of this potential, $a_{2} r_{2}^{-1} \cos \theta_{2}$, has the constant value $a_{2} / 2 R$ on the circle $r=R$, but is not constant on $r=1$. Similarly, mutatis mutandis, for $a_{1} r_{1}^{-1} \cos \theta_{1}$. The function $a_{1} r_{1}^{-1} \cos \theta_{1}+a_{2} r_{2}^{-1} \cos \theta_{2}$ will not, then, serve as the $\psi$ function, but it may be modified as follows.

We obtain the harmonic function $F(r, \theta)$ which, to within an additive constant, takes the values $-a_{1} r_{1}^{-1} \cos \theta_{1}$ on $r=R$, and $-a_{2} r_{2}^{-1} \cos \theta_{2}$ on $r=1$. Then the function

$$
\psi=a_{1} r_{1}^{-1} \cos \theta_{1}+a_{2} r_{2}^{-1} \cos \theta_{2}+F(r, \theta)+c,
$$

where $a_{1}, a_{2}$, and the available constant $c$ are chosen so that (3.1) and

$$
\psi\left(\infty^{\prime}\right)=\psi(\sqrt{R}, \pi)=0
$$

are satisfied, will be the imaginary part of the potential required.

Equations (3.1) and (3.3) are satisfied (see $\$ 7$ ) when

* When $(r, \theta)$ is the map of $z$ we shall use $\psi(z)$ and $\psi(r, \theta)$ indiscriminately where no misunderstanding can arise. The variable indicates the plane (and the point in the plane) at which the $\psi$ function is to be considered. Similarly for other functions with a physical significance. 


$$
\begin{aligned}
c & =-a_{1}\left(\frac{k^{\prime} \mathrm{K}}{2 \pi}+\frac{3}{4}\right)+\frac{a_{2}}{R}\left(\frac{\mathrm{K}}{2 \pi}-\frac{1}{4}\right), \\
a_{1} & =U^{2}\left(\frac{2 \mathrm{~K}(\alpha-\beta)-\pi(\alpha+\beta)}{\mathrm{K}\left(1+k^{\prime}\right)}\right), \\
\frac{a_{2}}{R} & =U^{2}\left(\frac{2 k^{\prime} \mathrm{K}(\alpha-\beta)+\pi(\alpha+\beta)}{\mathrm{K}\left(1+k^{\prime}\right)}\right) .
\end{aligned}
$$

(The apparent difference between the ways in which $a_{1}$ and $a_{2}$ appear in (3.4) is due to the fact that $a_{2}$ is on a circle of radius $R$.)

The function $F(r, \theta)$ in (3.2) was obtained in the form of a Fourier series:

$$
F(r, \theta)=\sum_{1}^{\infty}\left(A_{n} r^{n}+B_{n} r^{-n}\right) \cos n \theta .
$$

The conjugate harmonic function may then be written immediately as:

$$
-\sum_{1}^{\infty}\left(A_{n} r^{n}-B_{n} r^{-n}\right) \sin n \theta
$$

The conjugate function to

$$
a_{1} r_{1}^{-1} \cos \theta_{1}+a_{2} r_{2}^{-1} \cos \theta_{2} \text { is } \quad-a_{1} r_{1}^{-1} \sin \theta_{1}+a_{2} r_{2}^{-1} \sin \theta_{2},
$$

(the minus sign being due to the fact that $\theta_{1}$, as defined in Fig. 1(c), is measured in the negative sense). In this way the acceleration potential is obtained to within an additive constant $b$ (which disappears in evaluating the lift). Dropping the auxiliary coordinates, we have finally (see \$7)

$$
\begin{aligned}
& \text { on the fin: } \quad \varphi\left(R_{1} \theta\right)=\frac{-a_{2}}{2 R} \frac{\vartheta_{2}^{\prime}}{\vartheta_{2}}\left(\frac{1}{2} \theta, R\right)-\frac{a_{1}}{2} \frac{\vartheta_{4}^{\prime}}{\vartheta_{4}}\left(\frac{1}{2} \theta, R\right)+b \text {, } \\
& \text { on the flap: } \quad \varphi(1, \theta)=-\frac{a_{1}}{2} \frac{\vartheta_{1}^{\prime}}{\vartheta_{1}}\left(\frac{1}{2} \theta, R\right)-\frac{a_{2}}{2 R} \frac{\vartheta_{3}^{\prime}}{\vartheta_{3}}\left(\frac{1}{2} \theta, R\right)+b \text {; }
\end{aligned}
$$

in which $\vartheta_{i}^{\prime} / \vartheta_{i}\left(\frac{1}{2} \theta, R\right)$ is written for

$$
\frac{1}{\vartheta_{i}\left(\frac{1}{2} \theta, R\right)} \frac{d}{d\left(\frac{1}{2} \theta\right)}\left(\vartheta_{i}\left(\frac{1}{2} \theta, R\right)\right) .
$$

4. The lift and moment. From (1.2) we have $p=-\rho \varphi$. The lift on the air-foil at such a point as $G$ is given by

$$
l(z)=p(H)-p(G)=p\left(H^{\prime}\right)-p\left(G^{\prime}\right)=\rho[\varphi(\theta)-\varphi(-\theta)]=2 \rho(\varphi(\theta)-b)=l(\theta) .
$$

Thus, on the fin,

on the flap,

$$
l(R, \theta)=\rho\left(-\frac{a_{2}}{R} \frac{\vartheta_{2}^{\prime}}{\vartheta_{2}}\left(\frac{1}{2} \theta, R\right)-a_{1} \frac{\vartheta_{4}^{\prime}}{\vartheta_{4}}\left(\frac{1}{2} \theta, R\right)\right),
$$

$$
l(1, \theta)=\rho\left(-a_{1} \frac{\vartheta_{1}^{\prime}}{\vartheta_{1}}\left(\frac{1}{2} \theta, R\right)-\frac{a_{2}}{R} \frac{\vartheta_{3}^{\prime}}{\vartheta_{3}}\left(\frac{1}{2} \theta, R\right)\right)
$$


in which $a_{1}$ and $a_{2}$ are given by (3.4).

The total lift (on the flap, say) would then be given by

$$
L_{2}=\int_{1}^{1 / k^{\prime}} l(z) d z=\int_{0}^{\pi} l(\theta) \frac{d z}{d \theta} d \theta .
$$

The moment of the flap about the point $z=0$ is

$$
M_{2}(0)=\int_{1}^{1 / k^{\prime}} l(z) z d z
$$

and about any point $z_{1}$, it is

$$
M_{2}\left(z_{1}\right)=\int_{1}^{1 / k^{\prime}} l(z)\left(z-z_{1}\right) d z=M_{2}(0)-z_{1} L_{2} .
$$

The functions involved in these integrals are found in (4.2) and (2.2). It will be observed that the $\vartheta$-functions appearing have different parameters.

5. Results. The above integrations can be carried out exactly. The method is described in $\S 8$, and the results are:

$$
\begin{aligned}
\rho^{-1} U^{-2} L_{2}= & \frac{\alpha}{k^{\prime}}\left[\pi\left(1-k^{\prime}\right)+2 \mathrm{~K} k^{\prime}-\mathrm{E}\right]+\frac{\delta}{2 \pi k^{\prime}}\left(1-k^{\prime}\right)\left(\pi^{2}+4 \mathrm{~K}^{2} k^{\prime}\right), \\
\rho^{-1} U^{-2} M_{2}(0)= & \frac{\alpha}{k^{\prime 2}}\left(1-k^{\prime}\right)\left[2 \mathrm{E}-\frac{1}{2} \pi\left(1-k^{\prime}\right)\right] \\
& +\frac{\delta}{\pi k^{\prime 2}}\left[\pi\left(1-k^{\prime}\right)\left\{\mathrm{K} k^{\prime}+\mathrm{E}-\frac{1}{4} \pi\left(1-k^{\prime}\right)\right\}+\left(\mathrm{K} k^{\prime}-\mathrm{E}\right)^{2}\right], \\
\rho^{-1} U^{-2} L_{1}= & \frac{\alpha}{k^{\prime}}\left[2\left(\mathrm{E}-\mathrm{K} k^{\prime 2}\right)-\left(1-k^{\prime}\right)\left(2 \mathrm{~K} k^{\prime}-\pi\right)\right] \\
& +\frac{\delta}{2 \pi k^{\prime}}\left[\pi\left\{2 \mathrm{E}-\mathrm{K}\left(1+k^{\prime 2}\right)\right\}-\frac{1}{2}\left(1-k^{\prime}\right)\left(2 \mathrm{~K} k^{\prime}-\pi\right)(2 \mathrm{~K}+\pi)\right],
\end{aligned}
$$

where $L_{2}, M_{2}(0)$ have the meanings given in $\S 4, L_{1}$ is the total lift on the fin, $E$ is the complete elliptic integral of the second kind modulus $k$, and $\delta=\beta-\alpha$.

As a limiting case we consider the situation where the gap between fin and flap is so large in proportion to the chord of the fin (or flap) that they may be expected to act as independent airfoils. In this case $k^{\prime} \doteq 1, k \doteq 0$. Reference to tables of elliptic integrals (e.g., Jahnke, Emde, Tables of Functions, Teubner, 2nd Ed., 1938) or direct integration gives $K \doteq E \doteq \pi / 2$; so that, from (2.1) we have $R \doteq 0$. As an approximation, we neglect powers of $R$, and our formulae become (Whittaker, Watson, p. 489)

$$
\begin{gathered}
a_{1}=-2 \beta U^{2}, \quad a_{2} / R=2 \alpha U^{2}, \\
l(1, \theta)=2 \beta \rho U^{2} \cot \frac{1}{2} \theta .
\end{gathered}
$$

The expression in (5.2) is the usual one for the lift on a single straight wing with angle of attack $\beta$. For example: when the gap between fin and flap equals 8 times the chord of either, $R \doteq 0.0008$; and evaluating (4.2) we get, in this case, 


$$
l(1, \theta) \doteq \rho U^{2}(2.1 \beta-0.1 \alpha) \cot \frac{1}{2} \theta,
$$

which differs only slightly from (5.2) unless $|\beta-\alpha|$ is large; while for $\beta=\alpha$, (5.3) reduces to (5.2):

Some numerical examples are given in the table which follows. $C_{L}, C_{H}, C_{P}$ are the

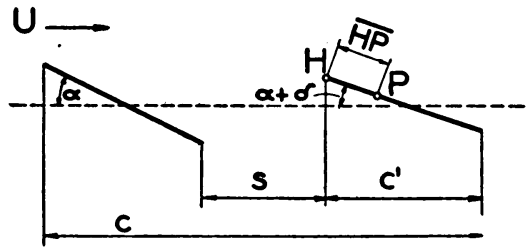

FIG. 2. usual lift and moment coefficients: lift coefficient, $C_{L}=L / \rho\left(U^{2} / 2\right) c, c$ being the total chord from leading edge of fin to trailing edge of flap; moment coefficient of the flap about its leading edge, $C_{H}=M_{2}(1) / \rho\left(U^{2} / 2\right) c^{2} ;$ moment coefficient of flap about $P, C_{P}=C_{H}-C_{L_{2}} \bar{H} \bar{P} / c$ where $\bar{H} \bar{P}$ is the distance from the leading edge of the flap to $P$ (see Fig. 2). The subscripts 1 and 2 indicate fin and flap, respectively, while $L$ indicates lift on either. In the table $\bar{H} \bar{P}$ is expressed as a fraction of $c^{\prime}$, the chord of the flap; and $\delta=\beta-\alpha$ and $\alpha$ are expressed in radians.

TABLE

( $\alpha$ and $\delta$ in radians)

\begin{tabular}{|c|c|c|c|c|c|}
\hline$s / c^{\prime}$ & 0.1 & 0.2 & 0.3 & 0.4 & 0.5 \\
\hline & \multicolumn{5}{|c|}{ Lift Coefficients } \\
\hline $\begin{array}{l}\text { Fin: } C_{L_{1}} \\
\text { Flap: } C_{L_{2}}\end{array}$ & $\begin{array}{l}4.57 \alpha+2.50 \delta \\
1.41 \alpha+2.05 \delta\end{array}$ & $\begin{array}{l}4.19 \alpha+2.05 \delta \\
1.51 \alpha+2.18 \delta\end{array}$ & $\begin{array}{l}3.88 \alpha+1.67 \delta \\
1.58 \alpha+2.22 \delta\end{array}$ & $\begin{array}{l}3.63 \alpha+1.42 \delta \\
1.61 \alpha+2.21 \delta\end{array}$ & $\begin{array}{l}3.41 \alpha+1.23 \delta \\
1.62 \alpha+2.18 \delta\end{array}$ \\
\hline$\overline{H P}$ & \multicolumn{5}{|c|}{ Moment Coefficients } \\
\hline $\begin{array}{l}0 ; C_{H} \\
0.1 c^{\prime} ; C_{.1} \\
0.2 c^{\prime} ; C_{.2} \\
0.25 c^{\prime} ; C_{.25}\end{array}$ & $\begin{array}{l}0.21 \alpha+0.28 \delta \\
0.15 \alpha+0.18 \delta \\
0.080 \alpha+0.080 \delta \\
0.046 \alpha+0.032 \delta\end{array}$ & $\begin{array}{l}0.20 \alpha+0.26 \delta \\
0.13 \alpha+0.16 \delta \\
0.065 \alpha+0.064 \delta \\
0.031 \alpha+0.015 \delta\end{array}$ & $\begin{array}{l}0.19 \alpha+0.25 \delta \\
0.12 \alpha+0.15 \delta \\
0.056 \alpha+0.058 \delta \\
0.022 \alpha+0.0094 \delta\end{array}$ & $\begin{array}{l}0.18 \alpha+0.24 \delta \\
0.12 \alpha+0.14 \delta \\
0.053 \alpha+0.052 \delta \\
0.017 \alpha+0.0060 \delta\end{array}$ & $\begin{array}{l}0.17 \alpha+0.22 \delta \\
0.11 \alpha+0.14 \delta \\
0.044 \alpha+0.049 \delta \\
0.012 \alpha+0.0048 \delta\end{array}$ \\
\hline
\end{tabular}

Finally, it might be of interest to note that one can locate a point about which the moment on the flap would be proportional to $\delta$. Although, if hinged at this point, the flap would not remain in the position $\delta=0$ without some restraint, still, when in this position, the moment on it would be zero. Thus if :

$$
\begin{array}{ll}
s / c^{\prime}=0.5, \quad \overline{H P}=0.270 c^{\prime}, & C_{.270}=-0.014 \delta ; \\
s / c^{\prime}=0.1, \quad \overline{H P}=0.313 c^{\prime}, & C_{.313}=-0.036 \delta .
\end{array}
$$

\section{MATHEMATICAL APPENDIX}

The following references will be used:

$\mathrm{W}=$ Whittaker and Watson, Modern Analysis, Cambridge, University Press, 4 ed., 1927.

$\mathrm{J}=\mathrm{Jahnke}$ and Emde, Tables of Functions, Leipzig, Teubner, 3 ed., 1938.

6. The mapping. (See §2.) The mapping is accomplished in two steps. First, we map the slit $z$-plane into the interior of a rectangle in the $\zeta$-plane by the function 


$$
\zeta=\int_{0}^{z}\left[\left(1-x^{2}\right)\left(1-k^{\prime 2} x^{2}\right)\right]^{-1 / 2} d x
$$

This rectangle has vertices at $\pm \mathrm{K}^{\prime}(k) \pm i \mathrm{~K}(k)$, the elliptic integrals being expressed as functions of the modulus $k$ complementary to $k^{\prime}$. The correspondence is: $z=1 \rightarrow \zeta=\mathrm{K}^{\prime}$, $z=k^{\prime-1} \rightarrow \zeta=\mathrm{K}^{\prime} \pm i \mathrm{~K}$, (plus, for the upper boundary of the cut), etc. The integral being multiply valued, the mapping is repeated infinitely of ten in the $\zeta$-plane, covering the plane without gaps by a net of congruent rectangles.

The second step of the mapping is

$$
w=\exp \left[\pi\left(\zeta-\mathbf{K}^{\prime}\right) / \mathrm{K}\right]
$$

This is periodic with period $2 i \mathrm{~K}$, hence $w$ takes both horizontal sides of the rectangle in to the part of the real $w$-axis between -1 and $-R,\left(R=\exp \left(-2 \pi \mathrm{K}^{\prime} / \mathrm{K}\right)\right)$, i.e., into the segment $D^{\prime} A^{\prime}$ (Fig. 1(c)). The vertical sides are mapped in to the circles $|w|=R$, $|w|=1$. Because of the periodicity of the exponential function, the full network of rectangles in the $\zeta$-plane is mapped into the ring $R \leqq|w| \leqq 1$, congruent points of different rectangles going in to the same point of the ring. Thus the mapping between the $z$ - and $w$-planes is one-to-one.

The inverse mapping is, with $w=r e^{i \theta},(\mathrm{W}, \mathrm{p} .492)$,

$$
z=\operatorname{sn}\left(\zeta, k^{\prime}\right)=\operatorname{sn}\left(\frac{\mathrm{K}}{\pi} \log w+\mathrm{K}^{\prime}, k^{\prime}\right)=\operatorname{sn}\left(\frac{\mathrm{K}}{\pi}(i \theta+\log r)+\mathrm{K}^{\prime}, k^{\prime}\right) .
$$

For the two important cases, $r=1, r=R$, the last formula reduces to (2.2) if we transform the elliptic function to one having a real argument (W, pp. 500-506). Note that when the elliptic function is expressed in terms of $\vartheta$-functions, the parameter, $q$, of the $\vartheta$-functions is $\exp \left(-\pi \mathrm{K}^{\prime} / \mathrm{K}\right)=R^{1 / 2}(\mathrm{~W}, \mathrm{p} .479$, ex. 3$)$.

7. The potential. (See §3.) The function $F(r, \theta)$, assumed as a Fourier series

$$
F(r, \theta)=\sum_{1}^{\infty}\left(A_{n} r^{n}+B_{n} r^{-n}\right) \cos n \theta
$$

must, to within an additive constant, satisfy the boundary conditions

$$
\begin{aligned}
& F(R, \theta)=-a_{1} r_{1}^{-1} \cos \theta_{1}=\frac{-a_{1}(1-R \cos \theta)}{1+R^{2}-2 R \cos \theta}, \\
& F(1, \theta)=-a_{2} r_{2}^{-1} \cos \theta_{2}=\frac{-a_{2}(R+\cos \theta)}{1+R^{2}+2 R \cos \theta},
\end{aligned}
$$

the expressions on the right being obtained from Fig. 1(c). But

$$
\begin{aligned}
& \frac{1-R \cos \theta}{1+R^{2}-2 R \cos \theta}=\Re \frac{1}{1-R e^{i \theta}}=\sum_{n=0}^{\infty} R^{n} \cos n \theta \\
& \frac{R+\cos \theta}{1+R^{2}+2 R \cos \theta}=\Re \frac{e^{-i \theta}}{1+R e^{-i \theta}}=\sum_{n=0}^{\infty}(-1)^{n} R^{n} \cos (n+1) \theta,
\end{aligned}
$$

where $\Re$ denotes the real part; and hence, substituting the last two expressions in (7.2) and comparing with (7.1), we get

$$
A_{n} R^{n}+B_{n} R^{-n}=-a_{1} R^{n}, \quad A_{n}+B_{n}=-a_{2}(-1)^{n-1} R^{n-1},(n \geqq 1),
$$


with solutions

$$
A_{n}=\frac{a_{1} R^{2 n}+a_{2}(-1)^{n} R^{n-1}}{1-R^{2 n}}, \quad B_{n}=-\frac{a_{1} R^{2 n}+a_{2}(-1)^{n} R^{3 n-1}}{1-R^{2 n}} .
$$

On substituting these values for $A_{n}, B_{n}$, or, more conveniently Eqs. (7.3), in the definition (7.1) of $F(r, \theta)$, we find

$$
F(R, \theta)=-a_{1} r_{1}^{-1} \cos \theta_{1}+a_{1}, \quad F(1, \theta)=-a_{2} r_{2}^{-1} \cos \theta_{2} ;
$$

so that $F(r, \theta)$ satisfies the required conditions. The constant $c$ in (3.2) must be chosen so that $\psi$ vanishes at the infinite point of the $z$-plane; i.e., (see (3.3)),

$$
\begin{aligned}
\psi\left(R^{1 / 2}, \pi\right)= & {\left[-a_{1} r_{1}^{-1} \cos \theta_{1}+a_{2} r_{2}^{-1} \cos \theta_{2}\right]_{r=R^{1 / 2}, \theta=\pi} } \\
& +\sum_{1}^{\infty}(-1)^{n}\left(A_{n} R^{1 / 2 n}+B_{n} R^{-1 / 2 n}\right)+c=0 .
\end{aligned}
$$

The value of the bracket is obtained from Fig. 1(c); and using (7.4) we find

$$
\begin{aligned}
c= & -\frac{a_{1}}{1+R^{1 / 2}}+\frac{a_{2}}{R^{1 / 2}\left(1-R^{1 / 2}\right)}+a_{1} \sum_{1}^{\infty}(-1)^{n} \frac{R^{3 n / 2}\left(1-R^{n}\right)}{1-R^{2 n}} \\
& -\frac{a_{2}}{R} \sum_{1}^{\infty} \frac{R^{3 n / 2}\left(1-R^{n}\right)}{1-R^{2 n}} .
\end{aligned}
$$

For the evaluation of the infinite sums in (7.6) we need certain formulae from the theory of elliptic functions. These are

$$
\begin{aligned}
& \sum_{1}^{\infty} \frac{q^{n}}{1+q^{2 n}}=\frac{\mathrm{K}}{2 \pi}-\frac{1}{4} \\
& \sum_{1}^{\infty} \frac{(-1)^{n} q^{n}}{1+q^{2 n}}=\frac{\mathrm{K} k^{\prime}}{2 \pi}-\frac{1}{4}
\end{aligned}
$$

Using $R=q^{2}$ (cf. end of $\S 6$ ), and making some obvious algebraic reductions, we find for $c$ the value given in (3.4).

The values of $a_{1}$ and $a_{2}$ are found from (3.1) which with (3.2) and (7.5) give the equations

$$
\frac{1}{2} a_{2} / R+a_{1}+c=U^{2} \alpha, \quad \frac{1}{2} a_{1}+c=U^{2} \beta .
$$

The solutions of these are contained in (3.4); and thus $\psi$ is completely determined.

The acceleration potential $\varphi$ is now obtained as the harmonic conjugate to the function $\psi$. We have, (see $\S 3$ )

$$
\varphi=-a_{1} r_{1}^{-1} \sin \theta_{1}+a_{2} r_{2}^{-1} \sin \theta_{2}-\sum_{1}^{\infty}\left(A_{n} r^{n}-B_{n} r^{-n}\right) \sin n \theta+b,
$$

where $b$ is an arbitrary constant; or, in terms of $r$ and $\theta$,

$$
\begin{aligned}
\varphi(r, \theta)= & \frac{-a_{1} r \sin \theta}{1+r^{2}-2 r \cos \theta}+\frac{a_{2} r \sin \theta}{R^{2}+r^{2}+2 r R \cos \theta} \\
& -\sum_{1}^{\infty}\left(A_{n} r^{n}-B_{n} r^{-n}\right) \sin n \theta+b .
\end{aligned}
$$


For the purpose of evaluating the lift we need only $\varphi(R, \theta)$ and $\varphi(1, \theta)$. These are given in (3.5); we establish the expression for $\varphi(1, \theta)$, that for $\varphi(R, \theta)$ being obtained in a similar way.

If, in (7.7), we set $r=1$, use (7.4), and collect terms we find

$$
\begin{aligned}
\varphi(1, \theta)= & -\frac{a_{1}}{2}\left\{\cot \frac{1}{2} \theta+4 \sum_{1}^{\infty} \frac{R^{2 n}}{1-R^{2 n}} \sin n \theta\right\} \\
& -\frac{a_{2}}{R}\left\{\frac{-R \sin \theta}{1+R^{2}+2 R \cos \theta}-\sum_{1}^{\infty}(-1)^{n} R^{n} \sin n \theta\right. \\
& \left.+2 \sum_{1}^{\infty} \frac{(-1)^{n} R^{n}}{1-R^{2 n}} \sin n \theta\right\}+b .
\end{aligned}
$$

But

$$
\frac{-R \sin \theta}{1+R^{2}+2 R \cos \theta}=\Im\left(\frac{R e^{-i \theta}}{1+R e^{-i \theta}}\right)=\sum_{1}^{\infty}(-1)^{n} R^{n} \sin n \theta,
$$

where $\Im$ denotes the imaginary part. Using this in (7.8) we obtain

$$
\begin{aligned}
\varphi(1, \theta)= & -\frac{a_{1}}{2}\left\{\cot \frac{1}{2} \theta+4 \sum_{1}^{\infty} \frac{R^{2 n}}{1-R^{2 n}} \sin n \theta\right\} \\
& -\frac{a_{2}}{2 R}\left\{4 \sum_{1}^{\infty} \frac{(-1)^{n} R^{n}}{1-R^{2 n}} \sin n \theta\right\}+b .
\end{aligned}
$$

Finally, the terms in the brackets in (7.9) may be expressed by means of $\vartheta$-functions. Making use of the results of W, p. 489, ex. 12, we get

$$
\varphi(1, \theta)=-\frac{a_{1}}{2} \frac{\vartheta_{1}^{\prime}}{\vartheta_{1}}\left(\frac{1}{2} \theta, R\right)-\frac{a_{2}}{2 R} \frac{\vartheta_{3}^{\prime}}{\vartheta_{3}}\left(\frac{1}{2} \theta, R\right)+b,
$$

as stated in (3.5). It is worth pointing out that the parameter of the $\vartheta$-functions met here is $R$, whereas in equation (2.2) the parameter is $R^{1 / 2}$.

8. Some definite integrals. (See $\S 4$.) The integrals in $\$ 4$ are combinations of the following eight integrals:

$$
\begin{aligned}
I_{m} & =\int_{0}^{\pi} \frac{\vartheta_{m}^{\prime}}{\vartheta_{m}}\left(\frac{1}{2} \theta, R\right) \frac{d z}{d \theta} d \theta, \\
J_{m} & =\int_{0}^{\pi} \frac{\vartheta_{m}^{\prime}}{\vartheta_{m}}\left(\frac{1}{2} \theta, R\right) z \frac{d z}{d \theta} d \theta, \quad(m=1,2,3,4),
\end{aligned}
$$

where $z$ and $\theta$ are connected by (2.2). For their evaluation we can make use of the following formulae:

$$
\begin{aligned}
& \frac{d}{d \theta} \frac{\vartheta_{1}^{\prime}}{\vartheta_{1}}\left(\frac{1}{2} \theta, R\right)=\frac{A z}{z-1}+B, \\
& \frac{d}{d \theta} \frac{\vartheta_{2}^{\prime}}{\vartheta_{2}}\left(\frac{1}{2} \theta, R\right)=\frac{A}{1-k^{\prime} z}+B,
\end{aligned}
$$




$$
\begin{aligned}
& \frac{d}{d \theta} \frac{\vartheta_{3}^{\prime}}{\vartheta_{3}}\left(\frac{1}{2} \theta, R\right)=\frac{A}{1+k^{\prime} z}+B, \\
& \frac{d}{d \theta} \frac{\vartheta_{4}^{\prime}}{\vartheta_{4}}\left(\frac{1}{2} \theta, R\right)=\frac{A z}{z+1}+B,
\end{aligned}
$$

where

$$
\begin{gathered}
z=\frac{1}{\mathrm{dn}(\mathrm{K} \theta / \pi, k)}, \\
A=-\mathrm{K}^{2} k^{2} / \pi^{2}, \quad B=\mathrm{K}(\mathrm{K}-\mathrm{E}) / \pi^{2} .
\end{gathered}
$$

We shall prove only (8.21), the others being established in exactly the same way.

From the quasi-periodic properties of the $\vartheta$-functions (W, p. 465 , ex. 4) we see that the left member of (8.21) is a doubly periodic function with periods $2 \pi, 2 \pi \tau$, where $\tau=2 i \mathrm{~K}^{\prime} / \mathrm{K}$. We may restrict ourselves to a single period rectangle, say the one having the origin as the southwest vertex. Here the left member of (8.21) is regular except for a pole with principal part $-2 / \theta^{2}$ (W, p. 466, p. 489, ex. 12, and the Laurent series for the cotangent). But, using (8.25) and W, p. 504.

$$
\begin{aligned}
z & =\left(1-\frac{k^{2}}{2} \frac{\mathrm{K}^{2} \theta^{2}}{\pi^{2}}+\cdots\right)^{-1}=1+\frac{k^{2}}{2 \pi^{2}} \mathrm{~K}^{2} \theta^{2}+\cdots, \\
\frac{A z}{z-1} & =\frac{2 A \pi^{2}}{k^{2} \mathrm{~K}^{2} \theta^{2}}\left(1+O\left(\theta^{2}\right)\right)=-\frac{2}{\theta^{2}}+\text { const. }+\cdots .
\end{aligned}
$$

Furthermore, $A z /(z-1)$ is regular at points in the period rectangle other than $\theta=0$, for $z$ is an elliptic function of order 2 and (8.3) shows that it takes the value 1 twice at $\theta=0$.

We see, therefore, that the difference

$$
\frac{d}{d \theta} \frac{\vartheta_{1}^{\prime}}{\vartheta_{1}}\left(\frac{1}{2} \theta, R\right)-\frac{A z}{z-1}
$$

is a doubly periodic function without singularities; thus it is a constant, $B$, which we evaluate at $\theta=\pi$. Using $\mathrm{W}$, p. 489 , ex. 12 , we have

and at $\theta=\pi$,

$$
\frac{d}{d \theta} \frac{\vartheta_{1}^{\prime}}{\vartheta_{1}}\left(\frac{1}{2} \theta, R\right)=-\frac{1}{2} \csc ^{2} \frac{1}{2} \theta+4 \sum_{1}^{\infty} \frac{n R^{2 n} \cos n \theta}{1-R^{2 n}} ;
$$

$$
\begin{aligned}
\frac{d}{d \theta} \frac{\vartheta_{1}^{\prime}}{\vartheta_{1}}\left(\frac{1}{2} \pi, R\right) & =-\frac{1}{2}+4 \sum_{1}^{\infty} \frac{(-1)^{n} n R^{2 n}}{1-R^{2 n}} \\
& =-\frac{1}{2}+2\left\{\sum_{1}^{\infty} \frac{(-1)^{n} n q^{2 n}}{1-q^{2 n}}-\sum_{1}^{\infty} \frac{(-1)^{n} n q^{2 n}}{1+q^{2 n}}\right\}, q=R^{1 / 2} .
\end{aligned}
$$

The sums are evaluated by reference to W, p. 535 , ex. 57 , and p. 512 , second formula differentiated. We obtain

$$
\frac{d}{d \theta} \frac{\vartheta_{1}^{\prime}}{\vartheta_{1}}\left(\frac{1}{2} \pi, R\right)=-\frac{\mathrm{K}^{2} k^{\prime}}{\pi^{2}}-\frac{\mathrm{KE}}{\pi^{2}} .
$$


Also, by (8.25), $z=k^{\prime-1}$ when $\theta=\pi$. Hence,

$$
B=\frac{\mathrm{K}}{\pi^{2}}\left[-\mathrm{K} k^{\prime}-\mathrm{E}+\mathrm{K} k^{2} k^{\prime-1}\left(k^{\prime-1}-1\right)^{-1}\right]=\frac{\mathrm{K}}{\pi^{2}}(\mathrm{~K}-\mathrm{E}), \quad\left(k^{2}+k^{\prime 2}=1\right),
$$

as in (8.26).

As before, we shall evaluate only one of the integrals, say $I_{1}$ in (8.11). This is accomplished by an integration by parts.

$$
\begin{aligned}
I_{1} & =\int_{0}^{\pi} \frac{\vartheta_{1}^{\prime}}{\vartheta_{1}}\left(\frac{1}{2} \theta, R\right) \frac{d z}{d \theta} d \theta \\
& =\left.\frac{\vartheta_{1}^{\prime}}{\vartheta_{1}}\left(\frac{1}{2} \theta, R\right) z(\theta)\right|_{0} ^{\pi}-\int_{0}^{\pi} \frac{d}{d \theta} \frac{\vartheta_{1}^{\prime}}{\vartheta_{1}}\left(\frac{1}{2} \theta, R\right) z(\theta) d \theta \\
& =-\left[\frac{\vartheta_{1}^{\prime}}{\vartheta_{1}}\left(\frac{1}{2} \theta, R\right)\right]_{\theta=0}-\int_{1}^{1 / k^{\prime}} \frac{d}{d \theta} \frac{\vartheta_{1}^{\prime}}{\vartheta_{1}}\left(\frac{1}{2} \theta, R\right) z \frac{d \theta}{d z} d z .
\end{aligned}
$$

In the extreme right member of (8.5) both the integrated part and the integral are infinite, but their difference, considered as the limit

is finite.

$$
\lim _{\epsilon \rightarrow 0}\left(-\left[\frac{\vartheta_{1}^{\prime}}{\vartheta_{1}}\left(\frac{1}{2} \theta, R\right)\right]_{\theta=\epsilon}-\int_{z(\epsilon)}^{1 / k^{\prime}} \frac{d}{d \theta} \frac{\vartheta_{1}^{\prime}}{\vartheta_{1}}\left(\frac{1}{2} \theta, R\right) z \frac{d \theta}{d z} d z\right)
$$

The integration can now be carried out without difficulty. In the integral in the right member of (8.5) use is made of (8.21), while $d \theta / d z$ is obtained from (8.25), the result being then expressed as a function of $z$. It is necessary to show that the singular contributions from the two terms of the right member of (8.5) cancel. This is done conveniently by employing their Laurent expansions. The integrals which present themselves are at worst elliptic integrals of the first two kinds, and can be found in $\mathrm{J}$, pp. 52-56. In this way we obtain the results of $\$ 5$. 